\title{
Kriterien erster und zweiter Ordnung für lokal beste Approximationen bei Problemen mit Nebenbedingungen
}

\author{
R. Hettich \\ Received February 10, 1975

\begin{abstract}
On First and Second Order Conditions
for Locally Best Approximations for Constraint Problems
\end{abstract}

Summary. In a previous paper [6] we considered the problem of approximating (in the Chebyshev-norm) a real-valued function $f(x)$ on a compact subset $X<\mathbb{R}^{m}, m \geq 1$, by an element of a set of functions $a(p, x), p \in P, P<\mathbb{R}^{n}$ an open set. Both necessary and sufficient conditions of the second order for an $a\left(p^{0}, x\right)$ to be a locally best approximation were derived. In this paper we generalize these results to problems where the set $P$ of admitted parameters is constrained by some inequality. Included are subjects as monotone, one-sided or restricted range approximation.

\section{Einleitung}

In [6] wurden Kriterien zweiter Ordnung für lokal beste Approximationen hergeleitet für Probleme der Art:

Man approximiere eine Funktion $f(x)$ durch eine Funktion aus der Menge $\left\{a(p, x) \mid p \in P_{0}\right\}$, mit einer offenen Parametermenge $P_{0}$, auf einem kompakten Teil des $\mathbb{R}^{m}$ bestmöglich im Sinne von Tschebyscheff. Die Funktionen $f$ und $a$ wurden dabei zweimal stetig differenzierbar vorausgesetzt.

In der vorliegenden Arbeit werden die in [6] gewonnenen Sätze auf Probleme ausgedehnt, bei denen der Parameterbereich $P$ noch durch Ungleichungen eingeschränkt wird: $P=\left\{p \in P_{0} \mid c(p, y) \leq 0, y \in Y\right\}$, mit einer kompakten Menge $Y$. Eine Reihe spezieller Probleme, wie die monotone und die einseitige Approximation, sind als Spezialfälle enthalten.

Neben Kriterien zweiter Ordnung behandeln wir auch solche erster Ordnung und setzen die Resultate mit bekannten Sätzen in Beziehung.

Im nächsten Abschnitt stellen wir einige häufige Bezeichnungen zusammen. Anschließend formulieren wir das Problem. Abschnitt 4 enthält einige Hilfssätze über lineare Ungleichungen, die wir später benötigen. Danach leiten wir Kriterien erster Ordnung ab und vergleichen sie mit bekannten Resultaten. Im sechsten Abschnitt beschäftigen wir uns mit Kriterien zweiter Ordnung, wobei wir vielfach von Ergebnissen von [6] Gebrauch machen werden. In Abschnitt 7 folgt ein einfaches Beispiel. Der letzte Abschnitt enthält einige Schlußbemerkungen. 


\section{Bezeichnungen}

Der Übersichtlichkeit halber seien hier einige der meistgebrauchten Bezeichnungen zusammengestellt. Es bezeichnet:

$x \quad$ einen Punkt in $\mathbb{R}^{m_{x}}$, meist $x \in X$

$x^{k} \quad$ die Punkte von $E_{x} \leqq X$

$y \quad$ einen Punkt in $\mathbb{R}^{m v}$, meist $y \in Y$

$y^{\prime} \quad$ die Punkte von $E_{y} \leqq Y$

$p, p^{0} \quad$ Punkte des Parameterbereichs $P_{0} \subseteq \mathbb{R}^{n}$

$p(t) \quad$ einen in $P_{0}$ verlaufenden Weg

$\xi, \eta \quad$ die erste bzw. zweite Ableitung von $p(t)$ in $t=0$

$f(x) \quad$ die zu approximierende Funktion

$a(p, x) \quad$ die Funktion, mit der approximiert wird

$c(p, y) \quad$ Funktion, die den zulässigen Teilbereich $P$ von $P_{0}$ festlegt: $P=\left\{p \in P_{\mathbf{0}} \mid c(p, y) \leq 0, y \in Y\right\}$

$e(p, x) \quad$ die Fehlerfunktion; $e(p, x)=|f(x)-a(p, x)|$

$g^{i}(x) \quad$ Funktionen, die $X$ definieren, $i \in I$

$h^{j}(x) \quad$ Funktionen, die $Y$ definieren, $j \in J$

$X \quad$ Bereich, in dem approximiert wird; $X \subseteq \mathbb{R}^{m_{x}}$, kompakt

$Y \quad$ Definitionsbereich der Nebenbedingungen, $Y \subseteq \mathbb{R}^{n_{\boldsymbol{y}}}$, kompakt

$P_{\mathbf{0}} \quad$ Menge der möglichen Parameter $p \in \mathbb{R}^{n}$

$P \quad$ Menge der zugelassenen Parameter

$E_{x} \quad$ Menge der Punkte von $X$, in denen $e\left(p^{0}, x\right)$ maximal ist

$E_{y} \quad$ Menge der Punkte von $Y$ mit $c\left(p^{0}, y\right)=0$

$\left.\begin{array}{l}I, I(x) \\ J, J(x)\end{array}\right\}$ endliche Indexmengen

Ableitungen bezeichnen wir folgendermaßen:

Ein unterer Index $x, y$ oder $p$ bezeichnet den Gradienten bzgl. $x, y$ oder $p: z$. B.

oder

$$
g_{x}^{i}(x)=\left(\frac{\partial g^{i}(x)}{\partial x_{1}}, \ldots, \frac{\partial g^{i}(x)}{\partial x_{m_{x}}}\right)^{T}
$$

$$
e_{p}(p, x)=\left(\frac{\partial e(p, x)}{\partial p_{1}}, \ldots, \frac{\partial e(p, x)}{\partial p_{n}}\right)^{T}
$$

(ein hochgestelltes $T$ bedeutet den Übergang zur transponierten Matrix).

Ein doppelter Index $x x, x p, p p, y y$ oder $y p$ bezeichnet die Matrizen der zweiten Ableitungen; z. B.

oder

$$
e_{x p}(p, x)=\left(\frac{\partial^{2} e(p, x)}{\partial x_{j} \partial p_{i}}\right), \quad j=1, \ldots, m_{x} ; \quad i=1, \ldots, n \quad\left(m_{x} \times n \text {-Matrix }\right)
$$

$$
h_{y y}^{j}(y)=\left(\frac{\partial^{2} h^{j}(y)}{\partial y_{i} \partial y_{k}}\right), \quad i, k=1, \ldots, m_{y} \quad\left(m_{y} \times m_{y} \text {-Matrix }\right) .
$$

Schließlich wollen wir vereinbaren, daß wir die Berechnung eines Funktionswertes für die Argumente $p=p^{0}, x=x^{k}$ bzw. $y=y^{l}$ durch einen hochgestellten Index $k$ bzw. $\ell$ und Weglassen der Argumente andeuten. Also etwa

$$
e_{x p}^{k}=e_{x p}\left(p^{0}, x^{k}\right) \text { oder } h^{i, \ell}=h^{l}\left(y^{\ell}\right) \text {. }
$$




\section{Formulierung des Problems}

Es seien $n, m_{x}, m_{y}$ natürliche Zahlen, $X$ und $Y$ kompakte Teilmengen von $\mathbb{R}^{m_{x}}$ resp. $\mathbb{R}^{m_{y}}$. Ferner sei $P_{\mathbf{0}} \leqq \mathbb{R}^{n}$ eine offene Menge und $a(p, x), c(p, y)$ in $P_{\mathbf{0}} \times X$ resp. $P_{0} \times Y$ stetige reellwertige Funktionen mit stetigen partiellen Ableitungen erster Ordnung nach den Komponenten $p_{1}, \ldots, p_{n}$ von $p$.

Hiermit sei

$$
P:=\left\{p \in P_{0} \mid c(p, y) \leq 0, y \in Y\right\} .
$$

Wir betrachten folgendes Approximationsproblem:

$Z \mathbf{u}$ einer gegebenen, auf $X$ stetigen Funktion $f(x)$ ist ein $p^{0} \in P$ zu bestimmen, so $\mathrm{da} B$

$$
\left\|f-a\left(p^{0}, .\right)\right\| \leq\|f-a(p, .)\|
$$

für alle $p$ im Durchschnitt einer Umgebung von $p^{0}$ mit $P .\|\cdot\|$ ist hierbei die Maximumnorm auf $X$. Ein $p^{0}$ mit obiger Eigenschaft nennen wir eine lokal beste Approximation. Gilt in (3.2) nur für $p=p^{0}$ Gleichheit, so sprechen wir von einer lokal besten Approximation im starken Sinn.

Bemerkung. Die Bedingung $c(p, y) \leq 0, y \in Y$, in (3.1) repräsentiert i. allg. unendlich viele Ungleichungen. Bei der einseitigen Approximation ist z.B. $Y=X$. Man könnte daran denken, anstatt mit $c: P_{0} \times Y \rightarrow \mathbb{R}$ mit einer Funktion $c$ : $P_{0} \times Y \rightarrow \mathbb{R}^{k}$ zu arbeiten. Sinnvoller wäre es jedoch, endlich viele Ungleichungen mit auf evtl. verschiedenen Bereichen $P_{0} \times Y_{i}$ definierten Funktionen $c_{i} z$ betrachten. Bei Randwertaufgaben könnte man z.B. neben Bedingungen im Gebiet noch solche auf dem Rand betràchten. Da die Erweiterung der folgenden Resultate auf diesen Fall auf der Hand liegt, wurde hierauf zugunsten einer einfacheren Bezeichnungsweise verzichtet.

Im folgenden betrachten wir stets ein festes $p^{0} \in P$ und stellen uns die Frage, wie man entscheiden kann, ob $p^{0}$ eine lokal beste Approximation ist oder nicht.

$\mathrm{Zu} p \in P$ definieren wir die Fehlerfunktion

$$
e(p, x)=|f(x)-a(p, x)|
$$

und setzen $\left\|e\left(p^{0},.\right)\right\|>0$ voraus. Ferner sei

und

$$
E_{x}=\left\{x \in X \mid e\left(p^{0}, x\right)=\left\|e\left(p^{0}, .\right)\right\|\right\}
$$

$$
E_{y}=\left\{y \in Y \mid c\left(p^{0}, y\right)=0\right\} \text {. }
$$

\section{Sätze über Ungleichungen}

Es sei $S$ eine Teilmenge des $\mathbb{R}^{n}$. Wir wollen die Lösbarkeit von Systemen von Ungleichungen

$$
x^{T} s \varrho 0, \quad s \in S,
$$

untersuchen, wo $\varrho$ für $<$ oder für $\leq$ steht. Je nachdem sprechen wir von einem strikten oder einem nichtstrikten System. $H(S)$ sei die konvexe, $K(S)$ die konische Hülle von $S$. Also

$$
\begin{aligned}
& H(S)=\left\{x=\sum_{i=1}^{r} u_{i} s_{i} \mid u_{i} \geq 0, \sum_{i=1}^{r} u_{i}=1, r<\infty\right\} \\
& K(S)=\{x=\lambda y \mid y \in H(S), \lambda \geq 0\} .
\end{aligned}
$$


Der erste Hilfssatz, der z. B. in [5] bewiesen wird, ist eine Verallgemeinerung des bekannten Lemmas von Farkas [2].

Hilfssatz 1. $K(S)$ sei abgeschlossen. Für jedes $d \in \mathbb{R}^{n}$ gilt genau eine der Aussagen (i) und (ii):

(i) Es ist $d \in K(S)$, d.h. es gibt endlich viele $s_{i} \in S, i=1, \ldots, r, r \geq 0$, und zugehörige $u_{i}>0$, so daß

$$
d=\sum_{i=1}^{r} u_{i} s_{i}
$$

(Für $r=0$ sei die rechte Seite in (4.3) der Nullvektor.)

(ii) Das folgende System ist lösbar:

$$
\begin{aligned}
& x^{T} s \leq 0, \quad s \in S \\
& x^{T} d>0 .
\end{aligned}
$$

Der folgende, ebenfalls in [5] bewiesene Hilfssatz gibt hinreichende Bedingungen dafür an, daß $K(S)$ abgeschlossen ist.

Hilfssatz 2. $K(S)$ ist abgeschlossen, wenn eine der Bedingungen (i) oder (ii) erfüllt ist:

(i) Die Menge $S$ ist endlich.

(ii) $S$ ist kompakt und $0 \notin H(S)$.

Es sei nun $S=\left\{s(y) \in \mathbb{R}^{n} \mid y \in \mathscr{Y}\right\}$, wo $\mathscr{Y}$ eine nichtleere, kompakte Teilmenge eines normierten Raumes und $s: \mathscr{Y} \rightarrow \mathbb{R}^{n}$ eine stetige Funktion sei.

Dann gilt folgende Verallgemeinerung des Transpositionssatzes von Gordan ([4]):

Hilfssatz 3. Genau dann ist das System von Ungleichungen

$$
x^{T} s(y)<0, \quad y \in \mathscr{Y}
$$

nicht lösbar, wenn es $y_{i} \in \mathscr{Y}$ und $u_{i}>0, i=1, \ldots, r, 1 \leq r \leq \operatorname{dim}(S)+1$, gibt, so daß

$$
\sum_{i=1}^{r} u_{i} s\left(y_{i}\right)=0 \text {. }
$$

Hierbei ist $\operatorname{dim}(S)$ die Dimension des von $S$ aufgespannten linearen Teilraums $\operatorname{des} \mathbb{R}^{n}$.

Beweis. Wegen der Stetigkeit von $s$ auf der kompakten Menge $\mathscr{Y}$ ist (4.4) genau lösbar, wenn das System

$$
\begin{aligned}
z & >0, \\
x^{T} s(y)+z & \leq 0, \quad y \in \mathscr{Y}
\end{aligned}
$$

eine Lösung $x, z(z \in \mathbb{R})$ besitzt. Sei $S^{*}=\left\{\left(\begin{array}{l}s \\ 1\end{array}\right) \in \mathbb{R}^{n+1} \mid s \in S\right\}$. Mit $S$ ist auch $S^{*}$ kompakt und ersichtlich gilt $0 \notin H\left(S^{*}\right)$. Nach Hilfssatz 2 ist $K\left(S^{*}\right)$ abgeschlossen, so $\mathrm{daB}$ wir Hilfssatz 1 anwenden können, welcher sofort die Behauptung liefert.

Definition. Wir wollen sagen, daß $S \leqq \mathbb{R}^{n}$ die Haarsche Bedingung erfüllt, wenn für je $k$ paarweise verschiedene $y_{1}, \ldots, y_{k} \in \mathscr{Y}, k \leq n$, die Vektoren

linear unabhängig sind.

$$
s\left(y_{1}\right), \ldots, s\left(y_{k}\right)
$$


Hilfssatz 4. $S=\{s(y) \mid y \in \mathscr{Y}\}$ erfülle die Haarsche Bedingung. Dann ist das strikte System

$$
x^{r} s<0, \quad s \in S,
$$

genau dann nicht lösbar, wenn das nichtstrikte nur die Lösung $x=0$ besitzt.

Beweis. Hat $x^{T} s \leq 0, s \in S$, nur die Lösung $x=0$, so ist klar, daß das strikte System nicht lösbar ist.

Sei umgekehrt das strikte System nicht lösbar. Wir nehmen an, $x_{0} \neq 0$ löse das nichtstrikte System.

Da aus $x_{0}^{T} s_{i}=0, i=1, \ldots, n, s_{i} \in S, s_{i}$ paarweise verschieden, wegen der Haarschen Bedingung $x_{0}=0$ folgen würde, gibt es höchstens $r \leq n-1$ Punkte

$$
y_{1}, \ldots, y_{r} \in \mathscr{Y} \text { mit } x_{0}^{T} s\left(y_{i}\right)=0 .
$$

Wegen der Haarschen Bedingung besitzt ferner $x^{T} s\left(y_{i}\right)=-1, i=1, \ldots, r$, eine Lösung $x=z$. Wegen der Stetigkeit von $s$ gibt es weiter zu jedem $y_{i}$ ein $\varepsilon_{i}>0$,

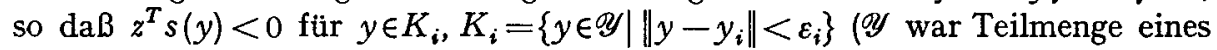
normierten Raumes).

Die Menge $\hat{\mathscr{Y}}=\mathscr{Y} \sim\left(\bigcup_{i=1}^{\mathscr{N}} K_{i}\right)$ ist kompakt. Daher existieren $m_{x}=$ $\max \left\{z_{0}^{T} s(y) \mid y \in \widehat{\mathscr{Y}}\right\}$ und $m_{0}=\max \left\{x_{0}^{T} s(y) \mid y \in \hat{\mathscr{Y}}\right\}$ und es ist $m_{0}<0$. Definiert man

$$
k=\left\{\begin{array}{cl}
1 & \text { für } m_{x} \leq 0 \\
-\frac{m_{0}}{2 m_{s}} & \text { für } m_{s}>0
\end{array},\right.
$$

so prüft man sofort nach, daß $x_{0}+k z$ eine Lösung des strikten Systems

$$
x^{T} s(y)<0, \quad y \in \mathscr{Y},
$$

ist, im Widerspruch zur Voraussetzung. Die Annahme $x_{\mathbf{0}} \neq 0$ ist also falsch und damit der Hilfssatz bewiesen.

Als nächstes beweisen wir eine Verallgemeinerung des Transpesitionssatzes von Stiemke [9]:

Hilfssatz 5. Sei $K(S)$ abgeschlossen. Genau dann hat das nichtstrikte System $x^{T} s(y) \leq 0, y \in \mathscr{Y}$, nur Lösungen mit $x^{T} s(y)=0, y \in \mathscr{Y}$, wenn es zu jedem $y_{0} \in \mathscr{Y}$ Punkte $y_{1}, \ldots, y_{r} \in \mathscr{Y}, r \leq \operatorname{dim}(S)$, und Zahlen $u_{i}>0$ gibt, so da $B$

$$
-s\left(y_{0}\right)=\sum_{i=1}^{r} u_{i} s\left(y_{i}\right)
$$

Beweis. $x^{T} s(y) \leq 0, y \in \mathscr{Y}$, besitze nur Lösungen mit $x^{T} s(y)=0, y \in \mathscr{Y}$. Für jedes $y_{0} \in \mathscr{Y}$ ist dann das System

$$
x^{T} s(y) \leq 0, \quad y \in \mathscr{Y}, \quad-x^{T} s\left(y_{0}\right)>0
$$

unlösbar. Nach Hilfssatz 1 folgt die Existenz von $y_{i}, u_{i}$, so daß (4.5) gilt.

Es gebe umgekehrt zu jedem $y_{0} \in \mathscr{Y}$ Punkte $y_{i}$ und $u_{i}>0$, so daB (4.5) gilt. Für jede Lösung $x$ von $x^{T} s(y) \leq 0, y \in \mathscr{Y}$, gilt dann $0 \leq-x^{T} s\left(y_{0}\right)=\sum_{i=1}^{r} u_{i} x^{T} s\left(y_{i}\right) \leq 0$, also $x^{T} s\left(y_{0}\right)=0$. Dies gilt für jedes $y_{0} \in \mathscr{Y}$ und der Hilfssatz ist bewiesen.

Schließlich benötigen wir den folgenden Hilfssatz über endliche inhomogene Systeme, der eine Spezialisierung des Satzes von Kuhn-Fourier (vgl. [10]) ist: 
Hilfssatz 6. Sei $M$ eine reelle $m \times n$-Matrix, $b \in \mathbb{R}^{m}$ ein Vektor. Dann ist das System $M x<b$ genau dann nicht lösbar, wenn es ein $u \in \mathbb{R}^{m}, u \geq 0, u \neq 0$, gibt mit $M^{T} u=0, u^{T} b \leq 0$.

\section{Kriterien erster Ordnung}

Sei $p^{0} \in P$ ein fester Punkt, $S=\left\{e_{p}\left(p^{0}, x\right) \mid x \in E_{x}\right\} \cup\left\{c_{p}\left(p^{0}, y\right) \mid y \in E_{y}\right\}$ und

$$
d_{S}=\operatorname{dim}(S) \text {. }
$$

Wir beginnen mit einer hinreichenden Bedingung erster Ordnung für lokal beste Approximationen im starken Sinn.

Satz 1. (a) Das System linearer Ungleichungen

$$
\xi^{T} e_{p}\left(p^{0}, x\right) \leq 0, \quad x \in E_{x}, \quad \xi^{T} c_{p}\left(p^{0}, y\right) \leq 0, \quad y \in E_{y}
$$

habe nur die Lösung $\xi=0$. Dann ist $p^{0}$ eine lokal beste Approximation im starken Sinn.

(b) Ist $d_{S}=n$ und $K(S)$ abgeschlossen, so ist die Bedingung in (a) der folgenden äquivalent:

$\mathrm{Zu}$ jedem $z \in E_{x}$ resp. $z \in E_{y}$ gibt es Punkte

$$
x^{j} \in E_{x}, \quad j=1, \ldots, r_{x}, \quad y^{j} \in E_{y}, \quad j=r_{x}+1, \ldots, r, \quad r \leq n,
$$

und Zahlen $u_{j}>0$, so daß

resp.

$$
e_{p}\left(p^{0}, z\right)+\sum_{j=1}^{r_{x}} u_{j} e_{p}^{j}+\sum_{j=r_{x}+1}^{r} u_{j} c_{p}^{j}=0
$$

$$
c_{p}\left(p^{0}, z\right)+\sum_{j=1}^{r_{x}} u_{j} e_{p}^{j}+\sum_{j=\gamma_{x}+1}^{r} u_{j} c_{p}^{j}=0 .
$$

Beweis. (b) folgt sofort aus Hilfssatz 5. Um (a) zu beweisen, zeigen wir, daß (5.1) eine Lösung $\xi \neq 0$ hat, wenn $p^{0}$ keine lokal beste Approximation im starken Sinn ist. Es gibt dann eine Folge $p_{k} \in P, p_{k} \neq p^{0}, k=1,2, \ldots$, mit $p_{k} \rightarrow p^{0}$, so da $B$

und

$$
e\left(p_{k}, x\right) \leq e\left(p^{0}, x\right), \quad x \in E_{z},
$$

$$
c\left(p_{k}, y\right) \leq c\left(p^{0}, y\right)=0, \quad y \in E_{y} .
$$

Wir setzen $p_{k}=p^{0}+t_{k} \xi_{k}$ mit $\xi_{k} \in \mathbb{R}^{n}$, $\left\|\xi_{k}\right\|_{2}=1\left(\|\cdot\|_{2}\right.$ bezeichne die euklidische Norm im $\mathbb{R}^{n}$ ) und $t_{k}>0$. Dann konvergieren die $\xi_{k}$ (evtl. gehe man zu einer Teilfolge über) gegen ein $\xi_{0},\left\|\xi_{0}\right\|_{2}=1$, und $t_{k} \rightarrow 0$. Aus (5.2) folgt für jedes $x \in E_{x}$

$$
e\left(p_{k}, x\right)-e\left(p^{0}, x\right)=t_{k} \xi_{k}^{T} e_{p}\left(p^{0}, x\right)+o\left(t_{k}\right) \leq 0 .
$$

Hieraus folgt aber $\xi_{0}^{T} e_{p}\left(p^{0}, x\right) \leq 0, x \in E_{x}$. Entsprechend folgt aus (5.3)

$$
\xi_{0}^{T} c_{p}\left(p^{0}, y\right) \leq 0, \quad y \in E_{y} .
$$

Also ist $\xi_{0}$ Lösung von (5.1) und $\xi_{0} \neq 0$ wegen $\left\|\xi_{0}\right\|_{\mathbb{2}}=1$. Damit ist Satz 1 bewiesen.

Wir zeigen weiter, daß die Unlösbarkeit des strikten Systems (5.1) notwendig dafür ist, daß $p^{0}$ eine lokal beste Approximation ist. 
Satz 2. $p^{0}$ sei eine lokal beste Approximation. Dann gelten die beiden folgenden äquivalenten Aussagen:

(a) Das folgende System besitzt keine Lösung $\xi$ :

$$
\xi^{T} e_{p}\left(p^{0}, x\right)<0, \quad x \in E_{x}, \quad \xi^{T} c_{p}\left(p^{0}, y\right)<0, \quad y \in E_{y} .
$$

(b) Es gibt $x^{j} \in E_{x}, j=1, \ldots, r_{x}, y^{j} \in E_{y}, \mathrm{j}=r_{x}+1, \ldots, r, 0 \leq r_{x} \leq r, 1 \leq r \leq d_{S}+1$, und Zahlen $u_{j}>0, \sum_{j=1}^{k} u_{j}=1$, so daB

$$
\sum_{j=1}^{r_{x}} u_{j} e_{p}^{j}+\sum_{j=\gamma_{x}+1}^{r} u_{j} c_{p}^{j}=0
$$

Berveis. Die Äquivalenz von (a) und (b) folgt aus Hilfssatz 3. Wir zeigen, daß (a) gilt. Wir nehmen an, $\xi_{0}$ sei eine Lösung von (5.4). Zu zeigen ist, daß $p^{0}$ keine Iokal beste Approximation ist. Da $e_{p}$ und $c_{p}$ stetig sind und $E_{z}, E_{y}$ kompakt, gibt es ein $\delta<0$ und ein $\varepsilon>0$, so daß mit

und

$$
E_{x \varepsilon}=\left\{x \in X \mid \text { es gibt } \tilde{x} \in E_{x} \text { mit }\|x-\bar{x}\|_{2}<\varepsilon\right\}
$$

gilt

$$
E_{y s}=\left\{y \in Y \mid \text { es gibt } \bar{y} \in E_{y} \text { mit }\|y-\bar{y}\|_{2}<\varepsilon\right\}
$$

$$
\begin{array}{ll}
\xi_{0}^{T} e_{p}(p, x) \leq \delta<0, & x \in \bar{E}_{x \varepsilon}, \\
\xi_{0}^{T} c_{p}(p, y) \leq \delta<0, & y \in \bar{E}_{y \varepsilon},
\end{array}
$$

für alle $p$ mit $\left\|p-p^{0}\right\|_{2} \leq \varepsilon$. Dabei sind $\bar{E}_{x \varepsilon}$ und $\bar{E}_{y_{\varepsilon}}$ die abgeschlossenen Hüllen von $E_{x \varepsilon}$ und $E_{y \varepsilon}$. Hier und im folgenden nehmen wir stillschweigend an, da $B$ alle auftretenden Parameter $p$ auf eine in $P_{0}$ enthaltene Umgebung von $p^{0}$ beschränkt sind. Da $P_{0}$ offen ist, ist dies keine wesentliche Einschränkung.

Die Mengen $X_{\varepsilon}=X \sim E_{x \varepsilon}$ und $Y_{\varepsilon}=Y \sim E_{y \varepsilon}$ sind kompakt. Daher gibt es $\varrho_{x}, \varrho_{y}$ und $\eta$, so daß für alle $p$ mit $\left\|p-p^{0}\right\|_{2} \leq \eta$ und $x \in X_{\varepsilon}, y \in Y_{\varepsilon}$ gilt

$$
e(p, x) \leq \varrho_{x}<\left\|e\left(p^{0}, .\right)\right\| \text { sowie } c(p, y) \leq \varrho_{y}<0 .
$$

Sei nun $p(t)=p^{0}+t \xi_{0}$. Für alle $x \in X$ gilt (vgl. z.B. [1], Th. 3.1)

$$
e(p(t), x)=e\left(p^{0}, x\right)+t \xi_{0}^{T} e_{p}(p(\theta), x), \quad 0<\theta<t .
$$

Es sei $t_{x}^{\prime}=\frac{\varepsilon}{\left\|\xi_{0}\right\|_{2}}$. Für $x \in \bar{E}_{x \varepsilon}$ gilt dann

$$
e(p(t), x) \leq\left\|e\left(p^{0}, .\right)\right\|+t \delta<\left\|e\left(p^{0}, .\right)\right\| \text { für } t \in\left(0, t_{x}^{\prime}\right] \text {. }
$$

Ist $x \in X_{\varepsilon}$, so gilt wegen (5.7) $e(p(t), x) \leq \varrho_{x}$ für alle $t$ mit $\left\|p(t)-p^{0}\right\|_{2} \leq \eta$. Hieraus folgt unmittelbar, daß es ein $t_{x}^{\prime \prime}>0$ gibt, so daß $e(p(t), x)<\left\|e\left(p^{0},.\right)\right\|$ für $x \in X_{\varepsilon}$, $t \in\left(0, t_{x}^{\prime \prime}\right]$. Somit gilt $e(p(t), x)<\left\|e\left(p^{0},.\right)\right\|$ für $x \in X$ und $t \in\left(0, t_{x}\right]$, wobei

$$
t_{x}=\min \left\{t_{x}^{\prime}, t_{x}^{\prime \prime}\right\}
$$

Völlig analog folgt aus (5.6) und (5.7) die Existenz von $t_{y}>0$, so daß

$$
c(p(t), y)<0 \text { für } y \in Y, t \in\left(0, t_{y}\right] .
$$

Damit wären aber $p(t)$ für $0<t \leq \min \left\{t_{x}, t_{y}\right\}$ bessere Approximationen als $p^{0}$, im Widerspruch zur Voraussetzung. Also ist Satz 2 bewiesen. 
Sichert man durch eine zusätzliche Bedingung (,, constraint qualification“) die Lösbarkeit des Teilsystems $\xi^{T} c_{p}\left(p^{0}, y\right)<0, y \in E_{y}$, von (5.4), so kann man in (b) $r_{x} \geq 1$ annehmen. Man erhält dann genau die Spezialisierung von Satz 1 in [7] auf das vorliegende Problem. Ebenfalls in [7] wird gezeigt, wie man von hier aus zu Alternantensätzen für Approximationsprobleme kommt, bei denen die zu approximierende Funktion und die Approximierende zwischen Schranken eingeschlossen sind.

Die Anwendung von Hilfssatz 4 auf Satz 1 und Satz 2 liefert uns ein notwendiges und hinreichendes Kriterium:

Satz 3. $S$ erfülle die Haarsche Bedingung. Dann sind die Bedingungen (a) und (b) von Satz 2 notwendig und hinreichend dafür, daB $p^{0}$ eine lokal beste Approximation im starken Sinn ist.

Ist $a(p, x)$ linear in den Variablen $p_{1}, \ldots, p_{n}$ und ist der Parameterbereich konvex, so gibt Satz 3 notwendige und hinreichende Kriterien sogar für global beste Approximationen. Da Satz 3 beste Approximationen im starken Sinn charakterisiert, folgt zugleich die Eindeutigkeit der besten Approximation in diesem Fall.

Hieraus kann man dann für eine Reihe verschiedener Typen von Approximationsproblemen Kriterien folgern, wie sie u.a. in [8] zu finden sind, insbesondere auch Alternantensätze.

Einen Úberblick über zahlreiche weitere Arbeiten auf dem Gebiet der Approximation mit Nebenbedingungen findet man in [11], eine Reihe von Anwendungen in [13].

Im Gegensatz zu Kriterien erster Ordnung, sind solche zweiter Ordnung, wie wir sie im nächsten Abschnitt herleiten wollen, unseres Wissens in der Literatur in expliziter Form nicht $z u$ finden.

\section{Kriterien zweiter Ordnung}

Wir setzen von nun an voraus, daß die Funktionen $f(x), a(p, x)$ und $c(p, x)$ nach allen Variablen zweimal stetig differenzierbar sind. Ferner benötigen wir zusätzliche Voraussetzungen über $X$ und $Y$ und die Funktionen $e\left(p^{0}, x\right)$ und $c\left(p^{0}, x\right) . p^{0} \in P$ ist dabei wieder ein fester Punkt. Zur Bezeichnungsweise verweisen wir auf Abschnitt 2.

(Ix). Es sei $X=\left\{x \in \mathbb{R}^{m_{x}} \mid g^{i}(x) \leq 0, i \in I\right\}$ mit einer endlichen Indexmenge $I$ und reellen, in $\mathbb{R}^{m_{x}}$ zweimal stetig differenzierbaren Funktionen $g^{i}(x)$. Jedem $x \in X$ ordnen wir die Indexmenge $I(x)=\left\{i \in I \mid g^{i}(x)=0\right\}$ zu. Für alle $x \in E_{x}$ seien dann die Vektoren $g_{x}^{i}(x), i \in I(x)$, linear unabhängig.

(Iy). Es sei $Y=\left\{y \in \mathbb{R}^{m_{y}} \mid h^{j}(y) \leq 0, j \in J\right\}$, wobei $J$, die $h^{j}(y)$ und die Vektoren $h_{y}^{j}(y), j \in J(y)=\left\{j \in J \mid h^{j}(y)=0\right\}$ identischen Voraussetzungen genügen wie $I$, die $g^{i}(x)$ und die $g_{x}^{i}(x)$ in $(\mathrm{Ix})$.

(IIx). Zu jedem $x^{k} \in E_{x}$ gebe es $v_{i}^{k}>0, i \in I\left(x^{k}\right)$, so daß gilt:

(i) $e_{x}^{k}-\sum_{i \in I\left(x^{k}\right)} v_{i}^{k} g_{x}^{i, k}=0$.

(ii) Auf $V_{k}=\left\{\zeta \in \mathbb{R}^{m_{x}} \mid \zeta^{T} g_{x}^{i, k}=0, i \in I\left(x^{k}\right)\right\}$ ist die quadratische Form

negativ definit.

$$
\zeta^{T} M_{k} \zeta, M_{k}=e_{x x}^{k}-\sum_{i \in I\left(x^{k}\right)} v_{i}^{k} g_{x x}^{i, k}
$$


(IIy). Zu jedem $y^{\ell} \in E_{y}$ gebe es $w_{j}^{\ell}>0, j \in J\left(y^{\ell}\right)$, so daß gilt:

(i) $c_{y}^{\ell}-\sum_{j \in J\left(y^{\ell}\right)} w_{j}^{\ell} h_{y}^{j, \ell}=0$.

(ii) Auf $W_{\ell}=\left\{\lambda \in \mathbb{R}^{m_{y}} \mid \lambda^{T} h_{y}^{j_{\ell} \ell}=0, j \in J\left(y^{\prime}\right)\right\}$ ist die quadratische Form

negativ definit.

$$
\lambda^{T} N_{\ell} \lambda, N_{\ell}=c_{y y}^{\ell}-\sum_{j \in J\left(y^{\ell}\right)} w_{j}^{\ell} h_{y y}^{j \ell},
$$

In [6] wurde gezeigt, da $\beta$ unter diesen Voraussetzungen $E_{x}$ und $E_{y}$ endlich sind:

$$
E_{x}=\left\{x^{1}, \ldots, x^{k x}\right\}, \quad E_{y}=\left\{y^{1}, \ldots, y^{k_{v}}\right\} .
$$

Sei nun $p(t)=p^{0}+t \xi+\frac{t^{2}}{2} \eta$ ein für $t \in\left[0, t^{*}\right], t^{*}>0$, ganz in $P_{0}$ verlaufender Weg. Dann wurde ebenfalls in [6] gezeigt, da $\mathrm{d}$ es unter den gemachten Voraussetzungen ein $t_{1} \in\left(0, t^{*}\right]$ gibt, so daß

$$
e(p(t), x) \leq \max _{x^{k} \in E_{x}}\left(e^{k}+t \xi^{T} e_{p}^{k}+\frac{t^{2}}{2}\left[\eta^{T} e_{p}^{k}+\xi^{T} e_{p p}^{k} \xi-\mu_{k}^{T} M_{k} \mu_{k}\right]\right)+o\left(t^{2}\right)
$$

für $x \in X$ und $t \in\left[0, t_{1}\right]$. Hierbei ist $M_{k}$ wie in (IIx) und $\mu_{k}$ ist eindeutig bestimmt durch das Gleichungssystem

$$
\left(\begin{array}{cc}
M_{k} & G_{k}^{T} \\
G_{k} & 0
\end{array}\right)\left(\begin{array}{c}
\mu_{k} \\
\sigma
\end{array}\right)=\left(\begin{array}{c}
-e_{x p}^{k} \xi \\
0
\end{array}\right)
$$

wo $G_{k}$ die Matrix ist mit den Zeilen $-\left(g_{x}^{i, k}\right)^{T}, i \in I\left(x^{k}\right)$.

$\mathrm{Da} c$ denselben Voraussetzungen wie $e$ genügt, gilt entsprechend: Es gibt ein $t_{2} \in\left(0, t^{*}\right]$, so daß

$$
c(p(t), y) \leq \max _{y^{\prime} \in E_{v}}\left(c^{l}+t \xi^{T} c_{p}^{l}+\frac{t^{2}}{2}\left[\eta^{T} c_{p}^{l}+\xi^{T} c_{p p}^{l} \xi-\nu_{l}^{T} N_{\ell} \nu_{l}\right]\right)+o\left(t^{2}\right)
$$

für $y \in Y$ und $t \in\left[0, t_{2}\right]$. Hierbei ist $N_{\ell}$ wie in (IIy) und $v_{\ell}$ ist eindeutig bestimmt durch das Gleichungssystem

$$
\left(\begin{array}{cc}
N_{\ell} & H_{\ell}^{T} \\
H_{\ell} & 0
\end{array}\right)\left(\begin{array}{l}
v_{\ell} \\
\tau
\end{array}\right)=\left(\begin{array}{c}
-c_{y p}^{\ell} \xi \\
0
\end{array}\right)
$$

wo $H_{\ell}$ die Matrix ist mit den Zeilen $-\left(h_{y}^{j, \ell}\right)^{T}, j \in J\left(y^{\ell}\right)$.

Aus (6.2) und (6.6) folgt, daß die $\mu_{k}$ und $\nu_{\ell}$ von $\eta$ unabhängig sind und von $\xi$ linear abhängen.

Sei nun $p^{0}$ eine lokal beste Approximation. Nach dem vorangehenden muß für beliebige $\xi, \eta \in \mathbb{R}^{n}, p(t)=p^{0}+t \xi+\frac{t^{2}}{2} \eta$ gelten:

Es gibt kein $\bar{t}>0$, so daß für $k=1, \ldots, k_{\lambda}, \ell=1, \ldots, k_{y}, t \in(0, \bar{t}]$ gilt

$$
e^{k}+t \xi^{T} e_{p}^{k}+\frac{t^{2}}{2}\left[\eta^{T} e_{p}^{k}+\xi^{T} e_{p p}^{k} \xi-\mu_{k}^{T} M_{k} \mu_{k}\right]<\left\|e\left(p^{0}, .\right)\right\|=e^{k}
$$

und

$$
t \xi^{T} c_{p}^{\ell}+\frac{t^{2}}{2}\left[\eta^{T} c_{p}^{\ell}+\xi^{T} c_{p p}^{\ell} \xi-v_{l}^{T} N_{l} v_{l}\right]<0
$$

Hieraus folgt, daß das System

$$
\xi^{T} e_{p}^{k}<0, k=1, \ldots, k_{x} ; \quad \xi^{T} c_{p}^{\ell}<0, \ell=1, \ldots, k_{y},
$$


keine Lösung hat. Nach Hilfssatz 3 ist dies genau dann der Fall, wenn es

$$
u_{x k} \geq 0, k=1, \ldots, k_{x} \text {, und } u_{y \ell} \geq 0, \ell=1, \ldots, k_{y} \text {, }
$$

gibt, so daB

$$
\sum_{k=1}^{k_{x}} u_{x k} e_{p}^{k}+\sum_{\ell=1}^{k_{y}} u_{y \ell} c_{p}^{\ell}=0
$$

Sei $\xi$ eine Lösung des Systems

$$
\xi^{T} e_{p}^{k} \leq 0, k=1, \ldots, k_{x} ; \quad \xi^{T} c_{p}^{\ell} \leq 0, \ell=1, \ldots, k_{y},
$$

mit $\xi^{T} e_{p}^{k}=0$ genau für $k=1, \ldots, k^{1}$ und $\xi^{T} c_{p}^{\ell}=0$ genau für $\ell=1, \ldots, k^{2}, k^{1}+k^{2}>0$. Dann gilt in (6.8) $u_{x k}=0, k=k^{1}+1, \ldots, k_{x}, u_{y \ell}=0, \ell=k^{2}+1, \ldots, k_{y}$, da andernfalls durch Multiplikation von (6.8) von links mit $\xi^{T}$ folgen würde

$$
0=\sum_{k=k^{1}+1}^{k_{x}} u_{x k} \xi^{T} e_{p}^{k}+\sum_{\ell=k^{2}+1}^{k_{y}} u_{y \ell} \xi^{T} c_{p}^{\ell}<0 .
$$

Für $k=k^{1}+1, \ldots, k_{x}$ resp. $\ell=k^{2}+1, \ldots, k_{y}$ sind (6.5) resp. (6.6) für hinreichend kleine $t$ erfüllt. Somit darf es kein $\bar{t}>0$ geben, so daß (6.5) und (6.6) mit $k^{1}, k^{2}$ an Stelle von $k_{x}, k_{y}$ erfüllt sind, wobei $\xi^{T} e_{p}^{k}=0$ und $\xi^{T} c_{p}^{\ell}=0$. Daher darf das folgende System von Ungleichungen keine Lösung $\eta$ besitzen:

$$
\begin{array}{ll}
\eta^{T} e_{p}^{k}<-\xi^{T} e_{p p}^{k} \xi+\mu_{k}^{T} M_{k} \mu_{k}, & k=1, \ldots, k^{1}, \\
\eta^{T} c_{p}^{\ell}<-\xi^{T} c_{p p}^{l} \xi+v_{\ell}^{T} N_{\ell} v_{\ell}, & \ell=1, \ldots, k^{2} .
\end{array}
$$

Mit Hilfssatz 6 folgt hieraus unmittelbar der folgende Satz.

Satz 4. Die Funktionen $f, a$ und $c$ seien zweimal stetig differenzierbar und die Voraussetzungen ( $\mathrm{Ix}$ ), (Iy), (IIx) und (IIy) seien erfüllt. Ist dann $p^{0}$ lokal beste Approximation, so gibt es zu jeder Lösung $\xi$ von (6.9) Zahlen $u_{x k} \geq 0, u_{y \ell} \geq 0$ (nicht alle Null), so daß (6.8) gilt und

$$
q(\xi)=\xi^{T}\left\{\sum_{k=1}^{k_{x}} u_{x k} e_{p p}^{k}+\sum_{\ell=1}^{k_{y}} u_{y \ell} c_{p p}^{\ell}\right\} \xi-\sum_{k=1}^{k_{x}} u_{x k} \mu_{k}^{T} M_{k} \mu_{k}-\sum_{\ell=1}^{k_{y}} u_{y \ell} \nu_{l}^{T} N_{\ell} v_{l} \geq 0 .
$$

$M_{k}$ und $N_{\ell}$ sind dabei wie in (IIx), (IIy), und die $\mu_{k}, \nu_{\ell}$ sind durch (6.2), (6.4) bestimmt. Wie erwähnt, hängen $\mu_{k}$ und $\nu_{\ell}$ linear von $\xi$ ab.

Wir zeigen weiter, daß eine nur wenig verschärfte Bedingung hinreichend ist.

Satz 5. Unter den Voraussetzungen von Satz 4 sei eine der beiden Bedingungen (a), (b) erfüllt:

(a) Das System (6.9) besitzt nur die Lösung $\xi=0$.

(b) $\mathrm{Zu}$ jeder Lösung $\xi$ von (6.9) gibt es $u_{x k} \geq 0, u_{y \ell} \geq 0$ (nicht alle Null), so daß (6.8) gilt und $q(\xi)>0$, falls $\xi \neq 0$.

Dann ist $p^{0}$ eine lokal beste Approximation im starken Sinn.

Berveis. Angenommen, es gebe eine Folge von Punkten $p^{i} \neq p^{0}, \lim _{i \rightarrow \infty} p^{i}=p^{0}$, mit $\left\|e\left(p^{i},.\right)\right\| \leq\left\|e\left(p^{0},.\right)\right\|$ und $p^{i} \in P$. Wir setzen $p^{i}=p^{0}+t_{i} \xi_{i}$ mit $t_{i}>0$ und $\left\|\xi_{i}\right\|=1$. Es gilt $\lim _{i \rightarrow \infty} t_{i}=0$. O.B.d.A. können wir annehmen, daß die Folge der $\xi_{i}$ konvergiert (evtl. gehe man zu einer Teilfolge über): $\lim _{i \rightarrow \infty} \xi_{i}=\xi,\|\xi\|=1$. 
Nach [6] Satz 1 gibt es unter den Voraussetzungen (Ix) und (II x) Umgebungen $U_{x^{3}, j}=1, \ldots, k_{x}$, der $x^{j}$ und ein $t_{0}>0$, so daß für $t \in\left[0, t_{0}\right] e\left(p^{0}+t \xi, x\right)$ in $X \frown \bigcup_{j=1}^{k_{x}} U_{x^{j}}$ lokale Maxima genau in den Punkten $x^{j}(t)=x^{j}+t \mu_{j}+\frac{t^{2}}{2} \lambda_{j}+o\left(t^{2}\right)$ besitzt, wo die $\mu_{j}$ durch (6.2) gegeben sind und die $\lambda_{j}$ dem Gleichungssystem

$$
\lambda_{j}^{T} g_{x}^{k, j}=-\mu_{j}^{T} g_{x x}^{k, j} \mu_{j}, \quad k \in I\left(x^{j}\right)
$$

genügen. Da $c$ entsprechenden Voraussetzungen genügt, gilt ebenso: Es gibt Umgebungen $U_{y \ell}, \ell=1, \ldots, k_{y}$, der $y^{\ell}$ und $t_{0}>0$ (man kann o.B.d.A. dasselbe $t_{0}$ wie oben wählen), so daß für $t \in\left[0, t_{0}\right] c\left(p^{0}+t \xi, y\right)$ in $Y \cap \bigcup_{\ell=1}^{k_{y}} U_{y^{\ell}}$ lokale Maxima genau in den Punkten $y^{l}(t)=y^{l}+t v_{\ell}+\frac{t^{2}}{2} \omega_{\ell}+o\left(t^{2}\right)$ besitzt, wo $\nu_{\ell}$ durch (6.4) bestimmt ist und die $\omega_{\ell}$ dem folgenden Gleichungssystem genügen:

$$
\omega_{\ell}^{T} h_{y}^{k, \ell}=-v_{\ell}^{T} h_{y y}^{k, \ell} \nu_{\ell}, \quad k \in J\left(y^{\ell}\right) .
$$

Aus der Annahme folgen die Ungleichungen

und

$$
\frac{1}{t_{i}}\left[e\left(p^{0}+t_{i} \xi_{i}, x^{j}\right)-e\left(p^{0}, x^{i}\right)\right] \leq 0, \quad j=1, \ldots, k_{x}
$$

$$
\frac{1}{t_{i}}\left[c\left(p^{0}+t_{i} \xi_{i}, y^{\ell}\right)-c\left(p^{0}, y^{\ell}\right)\right] \leq 0, \quad \ell=1, \ldots, k_{y} .
$$

Durch Grenzübergang $i \rightarrow \infty$ erhält man die Ungleichungen $\xi^{T} e_{p}^{j} \leq 0, j=1, \ldots, k_{x}$, $\xi^{T} c_{p}^{\ell} \leq 0, \ell=1, \ldots, k_{y}$, d.h. $\xi$ erfüllt (6.9). Ist Voraussetzung (a) erfüllt, hat man wegen $\|\xi\|=1$ sofort einen Widerspruch. Sei also (b) erfüllt und $u_{x j} \geq 0, u_{y \ell} \geq 0$ so, $\mathrm{daB}(6.8)$ gilt und $q(\xi)>0$. Für genügend große $i$ ist $t_{i} \in\left[0, t_{0}\right]$. Es gilt:

$$
\begin{aligned}
& 0 \geq \sum_{j=1}^{k_{x}} u_{x j}\left[e\left(p^{0}+t_{i} \xi_{i}, x^{j}\left(t_{i}\right)\right)-e\left(p^{0}, x^{j}\right)\right] \\
& +\sum_{\ell=1}^{k_{y}} u_{y \ell}\left[c\left(p^{0}+t_{i} \xi_{i}, y^{\ell}\left(t_{i}\right)\right)-c\left(p^{0}, y^{\ell}\right)\right] \\
& =t_{i} \xi_{i}^{T} \underbrace{\left[\sum_{j=1}^{k_{x}} u_{x j} e_{p}^{j}+\sum_{\ell=1}^{k_{y}} u_{y \ell} c_{p}^{\ell}\right]}_{=0 \operatorname{nach}(6.8)} \\
& +t_{i} \underbrace{\left[\sum_{j=1}^{k_{x}} u_{x j} \mu_{j}^{T} e_{x}^{i}+\sum_{\ell=1}^{k_{y}} u_{y \ell} \nu_{l}^{T} c_{y}^{\ell}\right]} \\
& =0 \text { nach (IIx), (6.2) und (IIy), (6.4) } \\
& +\frac{t_{i}^{q}}{2}\left\{\xi_{i}^{T}\left[\sum_{j=1}^{k_{x}} u_{x j} e_{p p}^{j}+\sum_{i=1}^{k_{y}} u_{y \ell} c_{p p}^{l}\right] \xi_{i}\right. \\
& +2 \xi_{i}^{r}\left[\sum_{j=1}^{k_{x}} u_{x j} e_{p x}^{j} \mu_{j}+\sum_{\ell=1}^{k_{y}} u_{y \ell} c_{p y}^{l} \nu_{\ell}\right] \\
& +\sum_{j=1}^{k_{x}} u_{x j}\left[\lambda_{j}^{T} e_{x}^{j}+\mu_{j}^{T} e_{x x}^{j} \mu_{j}\right] \\
& \left.+\sum_{\ell=1}^{k_{y}} u_{y \ell}\left[\omega_{l}^{T} c_{y}^{\ell}+v_{l}^{T} c_{y y}^{\ell} \nu_{l}\right]\right\}+o\left(t_{i}^{2}\right) .
\end{aligned}
$$


Nach (IIx), (II y), (6.11) und (6.12) erhält man für die beiden letzten Summen

$$
\sum_{\ell=1}^{k_{x}} u_{x j} \mu_{j}^{T} M_{j} \mu_{j} \quad \text { resp. } \quad \sum_{j=1}^{k_{y}} u_{y \ell} \nu_{\ell}^{T} N_{\ell} v_{\ell} .
$$

Beachtet man noch, daß durch Multiplikation von (6.2) von links mit $\left(\begin{array}{c}\mu_{j} \\ \sigma\end{array}\right)^{T}$ und von (6.4) mit $\left(\begin{array}{c}\nu_{\ell} \\ \tau\end{array}\right)^{T}$ die Relationen $\mu_{j}^{T} M_{j} \mu_{j}=-\mu_{i}^{T} e_{x p}^{i} \xi \operatorname{resp} . \nu_{l}^{T} N_{\ell} \nu_{\ell}=-v_{\ell}^{T} c_{y p}^{l} \xi$ folgen, so ergibt sich durch Grenzübergang $i \rightarrow \infty q(\xi) \leq 0$ im Widerspruch zur Voraussetzung. Die Annahme war daher falsch und Satz 5 ist bewiesen.

Bemerkung. Beim Beweis von Satz 3 in [6] wurde stillschweigend angenommen, daß sich $t_{0}$ in Abhängigkeit von $\xi$ stetig wählen läßt, was durch einen umständlichen, wenn auch im einzelnen elementaren Beweis nachzuweisen wäre. Deshalb haben wir hier eine andere Beweistechnik vorgezogen und damit zugleich einen neuen Beweis von Satz 3 in [6] gegeben, der ohne eine solche Prämisse auskommt.

\section{Ein Beispiel}

Zur Illustration behandeln wir folgendes einfache Beispiel: Im Intervall $\left[-\frac{1}{2}, 1\right]$ soll die Funktion $f(x) \equiv 0$ durch eine Funktion

$$
a(p, x)=-\frac{1}{2} p^{2}+2 p x-x^{2}, \quad p \in \mathbb{R},
$$

einseitig von unten approximiert werden, d.h. unter der Nebenbedingung

$$
a(p, x) \leq 0, \quad x \in\left[-\frac{1}{2}, 1\right] .
$$

Wir zeigen, da $\beta p^{0}=0$ eine lokal beste Approximation ist (wie man leicht sieht, sogar die global beste Approximation).

Es wird $E_{x}=\{1\}, E_{y}=\{0\}$. Wegen $e_{p}(0,1)=-2$ und $c_{p}(0,0)=a_{p}(0,0)=0$ wird System (6.9) einfach

$$
\begin{aligned}
-2 \xi & \leq 0 \\
0 \xi & \leq 0, \quad \text { also } \xi \geq 0 .
\end{aligned}
$$

Ersichtlich gilt (6.8) für $u_{x 1}=0\left(k_{x}=1=k_{y}\right)$ und beliebige $u_{y}=u_{y 1}>0$. Ferner rechnet man leicht nach, daß (IIy) (ebenso wie (Ix), (Iy) und (IIx)) erfüllt ist und

$$
N=c_{y y}(0,0)=-2 \text {. }
$$

(6.4) reduziert sich damit auf

$$
-2 \nu=-c_{y p}(0,0) \xi=-2 \xi, \quad \text { d.h. } \nu=\xi .
$$

Mit $c_{p p}(0,0)=-1$ folgt schließlich

$$
q(\xi)=\xi\left(-u_{y}+2 u_{y}\right) \xi=u_{y} \xi^{2}>0 .
$$

für $u_{y}>0, \xi>0$. Nach Satz 5 ist damit gezeigt, daß $p^{0}=0$ eine lokal beste Approximation sogar im starken Sinne ist.

Wir bemerken weiter, daß die Punkte von $E_{x}$ und $E_{y}$ keine $H_{1}$-Menge zu $p^{0}=0$ bilden ([13], S. 102), so daß die dort angeführte hinreichende Bedingung nicht 
erfüllt ist. Wäre $E_{x} \cup E_{y}$ eine $H_{1}$-Menge, so dürfte es kein $p$ geben mit

und

$$
a(p, 1)-a(0,1)>0
$$

$$
a(p, 0)-a(0,0) \leq 0 \text {. }
$$

Für $p=1$ erhält man aber $z$. B. $a(p, 1)-a(0,1)=3 / 2$ und $a(p, 0)-a(0,0)=-1 / 2$.

\section{Schlußbemerkungen}

Während wir zur Herleitung der Kriterien erster Ordnung außer Stetigkeitsund Differenzierbarkeitseigenschaften keine Voraussetzungen benötigten, wurden die Kriterien zweiter Ordnung unter zusätzlichen Bedingungen (Ix), (Iy), (IIx) und (IIy) abgeleitet. In der Praxis werden sich diese im konkreten Fall i. allg. leicht prüfen lassen.

Macht man von den in [5] (und früher in [12]) abgeleiteten Kriterien für Optimierungsprobleme mit endlich oder unendlich vielen Nebenbedingungen Gebrauch, so kommt man auch mit schwächeren Voraussetzungen aus, die dann aber i. allg. schwieriger zu prüfen sind. Der Weg über Optimierungsprobleme wird auch in [3] gewählt, um Kriterien erster Ordnung für Approximationsprobleme mit (z.B.) Interpolationsbedingungen abzuleiten. Um solche Aufgaben zu behandeln, wäre es sinnvoll, bei der Definition von $P$ auch Gleichungen als Nebenbedingungen zuzulassen, was ohne weiteres möglich ist.

\section{Literatur}

1. Afriat, S. N.: Theory of maxima and the method of Lagrange. SIAM J. Appl. 20, $343-357$ (1971)

2. Farkas, J.: Uber die Theorie der einfachen Ungleichungen. J. Reine Angew. Math. 124, 1-27 (1901)

3. Gehner, K. R.: Necessary and sufficient optimality conditions for the Fritz John problem with linear equality constraints. SIAM J. Control, 12, 140-149 (1974)

4. Gordan, P.: Über die Auflösung linearer Gleichungen mit reellen Coefficienten. Math. Ann. 6, 23-28 (1873)

5. Hettich, R. : Extremalkriterien für Optimierungs- und Approximationsaufgaben, Technische Hogeschool Twente, Enschede, Dissertation 1973

6. Hettich, R.: Kriterien zweiter Ordnung für lokal beste Approximationen. Num. Math. 22, 409-417 (1974)

7. Hoffmann, K. H.: Nichtlineare Tschebyscheff-Approximation mit Nebenbedingungen: Anwendungen, Numer. Math. 14, 383-393 (1970)

8. Lewis, J. T.: Approximation with convex contrains. SIAM Rev., Vol. 15, 193-217 (1973)

9. Stiemke, E.: Über positive Lösungen homogener linearer Gleichungen. Math. Ann. 76, 340-342 (1915)

10. Stoer, J., Witzgall, Ch.: Convexity and optimization in finite dimensions I. Berlin-Heidelberg-New York: Springer 1970

11. Taylor, G. D.: Uniform approximation with side conditions. In: G. G. Lorentz (ed.), Approximation theory. New York-London: Academic Press, p. 495-503, 1973 
12. Wetterling, W.: Definitheitsbedingungen für relative Extrema bei Optimierungsund Approximationsaufgaben. Numer. Math. 15, 122-136 (1970)

13. Collatz, L., Krabs, W.: Approximationstheorie. Teubner Studienbücher Mathematik, Stuttgart: Teubner, 1973

R. Hettich

Technische Hogeschool Twente

Onderafdeling der Toegepaste Wiskunde

Postbus 217

Enschede

Niederlande 